\title{
Policy-Combination Oriented Optimization for Public Transportation Based on the Game Theory
}

\author{
Wentao Zhu $(\mathbb{D})^{1}$ Mengwei Chen, ${ }^{1}$ Dianhai Wang $\mathbb{D},{ }^{1}$ and Dongfang Ma $\mathbb{D}^{2}$ \\ ${ }^{1}$ College of Civil Engineering and Architecture, Zhejiang University, Hangzhou, Zhejiang 310058, China \\ ${ }^{2}$ Institute of Marine Information Science and Technology, Zhejiang University, Hangzhou, Zhejiang 310058, China \\ Correspondence should be addressed to Dianhai Wang; wangdianhai@zju.edu.cn
}

Received 3 November 2017; Revised 13 June 2018; Accepted 5 July 2018; Published 17 July 2018

Academic Editor: Bruno G. M. Robert

Copyright (C) 2018 Wentao Zhu et al. This is an open access article distributed under the Creative Commons Attribution License, which permits unrestricted use, distribution, and reproduction in any medium, provided the original work is properly cited.

\begin{abstract}
This research aims at detecting the interactions between policy maker and travelers when making public transport policy and strategies to optimize relevant policy combination. In the two scenarios of whether to set a bus lane or not, travel cost functions of bus and car are proposed, respectively, with the in-vehicle comfort level of passengers considered. By introducing the bottleneck model and transit assignment model, travelers' behaviors are revealed according to different travel mode. Focusing on minimizing the total cost of the system (TSC), Stackelberg game model is built to describe the dynamic interactions between the government, the bus company, and travelers. Finally, kriging surrogate method is proposed on account of numerical simulation to find solution to the game model and propose the optimal policy combination and resource allocation. The results show an effective performance: under the assumption that the travel distance is $20 \mathrm{~km}$, the optimized policy combination can reduce TSC by $8.59 \%$ and $9.82 \%$ in two scenarios, respectively, and reduce travel cost per person by $10.28 \%$ and $15.85 \%$, respectively.
\end{abstract}

\section{Introduction}

The rapid pace of economic development and the explosive growth in car ownership has contributed to urban congestion. Public transportation with its strength of high capacity and low emission has become important in mitigating traffic congestion. Thus, public transit priority policy is widely accepted. However, the development of urban public transit is not so promising for the slow increase or even decrease in some regions; in the passenger volume, it has transferred. One of the reasons is that the answer to the question of "to what extent have the policies had impact on travelers in the complicated traffic environment" stays unclear. Besides, there is a lack of necessary technical criterion on whether or when the policy implementations should be taken, and how much strength should be put. Therefore, to choose an appropriate policy combination to enhance the benefit of the whole traffic system based on various relevant factors to meet the needs of its sustainable development is a critical problem in the transportation field.

To attract citizens to travel by public transport instead of private cars to ease the pressure of congestion, policy makers have made some effort on policy implementations in two ways. On the one hand, the use of public transport is encouraged by improving the level of service and reducing the cost of travel in terms of public transport pricing, providing public transport subsidy and setting bus lane and signal priority. On the other hand, the use of private cars is reduced by carrying out parking charge, setting restrictions for carpurchasing and restriction policy of private car use based on even-and-odd numbered license plate, implementing road pricing and so forth. Up to now, there is massive research on either the policy mentioned previously based on the investigation of travel cost determined by people's travel choice. The choices were made under the assumption that every individual would choose the mode of travel with the maximum utility for himself. Yet the traffic system contains not only travelers, but also sectors such as the bus companies and the government. While making strategies, the connection between these parts and their conflicts of benefit should be taken into consideration. In view of this, the paper uses the game theory to better explain how these groups make decisions.

Game theory is an effective tool for system analysis. It focuses on the study of the dependence and influence 
between decision-making bodies and the equilibrium results related. In the field of transportation, the game theory is mainly used to detect the relationship among travelers, between travelers and the government, and among operators. Of all these three relationships, the first one is often modelled by symmetry game which means all the travelers have the same aim and use the same travel strategy. Vickrey [1] proposed classical bottleneck model on the basis of queuing theory to describe traveler's choice of departure time in peak hours which is essentially the Nash equilibrium of the game between travelers when they choose their departure time. In the model, it is supposed that there is only one freeway with limited capacity between home and work that every commuter must pass. Everyone hopes to get to the destination on time but the limited capacity of the bottleneck does not allow so. As the consequences, there are commuters arriving at work place earlier or later than the time they are supposed to arrive, generating relevant cost for the delay. This pushes the travelers to select a best departure time to minimize their travel cost considering their travel time and the delay. Later on, Yang, Huang [2], Wu [3, 4], and Gonzales E J [5] et al. (Xiao et al. [6]; Amirgholy et al. [7]; DePalma et al. [8]; Li et al. [9]) expended the analysis from different perspectives by adding new routes as options for travelers, considering the travel mode competition of bus and car, altering the travelers' attributes and so forth to discuss problems related to road charging, park and ride, and public transport pricing. However, these models mainly explain the base-level equilibrium between travelers, missing the role of decision-maker. Furthermore, various research used classic time-cost function to calculate travel cost, ignoring the cost of passengers' in-vehicle comfort which is inaccurate. In terms of the game between travelers and decision-maker, they often have different objective function and follow the Leader-Follower principle. Taking the widely used Stackelberg model (Berglund et al. [10]; Wang et al. [11]) as an example, it suggests the different levels for participants in the game and sets the decision-maker as the leader and travelers as the followers. Zuylen et al. [12], Lim et al. [13], and Hollander et al. [14] adopt this model to seek solution for problems towards urban road control, road network design, parking policy, and so forth. Even so, LeaderFollower model is a very hard mathematical problem of bilevel programming. Pang et al. [15] point out that although the model can be regarded as Mathematical Programming with Equilibrium Constraints (MFEC) or Quasi-Variational Inequality Problem (QVIP) which is easy to understand, the theory and algorithm are complicated, especially when there is uncertainty in the constraints.

In traffic system, any traffic state is the result of gaming equilibrium of all participants with different travel modes based on how the decision-makers allocate the resource. This kind of equilibrium is built upon the fact that every participant pays the least cost for traveling. Because of the correlation between policies, unwise combination of policies may weaken every policy's effect on the traffic system. Most existing research just focuses on analyzing single policy optimization because as the model gets more complicated, the dimensions of the policy variable increased and it is hard to find a solution. Nonetheless, in the real world, several traffic policies added up together are commonly seen which lack studies for their interactions. This makes it necessary to propose modelling analysis for strategies such as parking charges, road pricing, public transport pricing and subsidy, bus lane setting, and so forth and take the connection between policies and decision-making for all transportation participants into consideration, to conduct synergic optimization for the arrangement of resources and policies.

This paper builds up a cost function considering two travel modes of bus and car on account of two scenarios of having bus lane or not. By using bottleneck model and transit assignment model, travelers' choices of different modes in system equilibrium are discussed. Furthermore, the dynamic interaction among the government, bus company, and travelers in the system is depicted with Stackelberg game model. Finally, kriging surrogate model is adopted to integrate policies and resources in the system and optimize them. The objective is to solve three problems: (1) whether to implement a certain policy and how hard; (2) how travelers would change their behaviors after the new policy is put into practice; and (3) how to improve the policy combination to reach system optimal.

Before introducing the research, it is necessary to review the basic idea of Stackelberg game model which is as follows [16]. One of the players (the leader) has the privilege to impose his strategy before the other player (the follower). Let $\Gamma_{L}$ and $\Gamma_{F}$ be the strategy for leader and follower, respectively, and $\gamma_{L} \in \Gamma_{L}, \gamma_{F} \in \Gamma_{F}$. Let $J_{L}\left(\gamma_{L}, \gamma_{F}\right)$ and $J_{F}\left(\gamma_{L}, \gamma_{F}\right)$ be their payoff functions, then for any existing $\gamma_{L}$ suggested by leader, follower would give a $\gamma_{F}^{0} \in \Gamma_{F}$ to maximize his own payoff $J_{F}$. Knowing follower's strategy $J_{F}$, leader would come up with a strategy (taking followers reaction into consideration) so that he get the maximum payoff. Thus, follower's reaction can be described as

$$
\begin{aligned}
& R\left(\gamma_{L}\right) \\
& \quad=\left[\gamma_{F}^{0} \in \Gamma_{F}: J_{F}\left(\gamma_{L}, \gamma_{F}^{0}\right) \geq J_{F}\left(\gamma_{L}, \gamma_{F}\right), \forall \gamma_{F} \in \Gamma_{F}\right]
\end{aligned}
$$

And the leader's choice is

$$
\inf J_{L}\left(\gamma_{L}^{s}, \gamma_{F}\right) \geq \inf J_{L}\left(\gamma_{L}, \gamma_{F}\right), \quad \forall \gamma_{L} \in \Gamma_{L}
$$

Both players need to address their strategy at the beginning of the game and leader has the priority to declare first.

The subsequent parts of the paper are arranged in four parts. The second part puts forward the cost function of both bus and car travel considering the in-vehicle comfort under the influence of various transport policies and employs bottleneck model to examine the Nash equilibrium-based travel cost of travelers which is the follower. The third part scrutinizes the revenue function of the government, the bus company, and travelers and suggests Stackelberg game model for transport policy-combination oriented optimization. Part 4 tries to give optimized solution to the game model and verify the feasibility and effectiveness of the methodology used in the paper by conducting numerical simulation based 


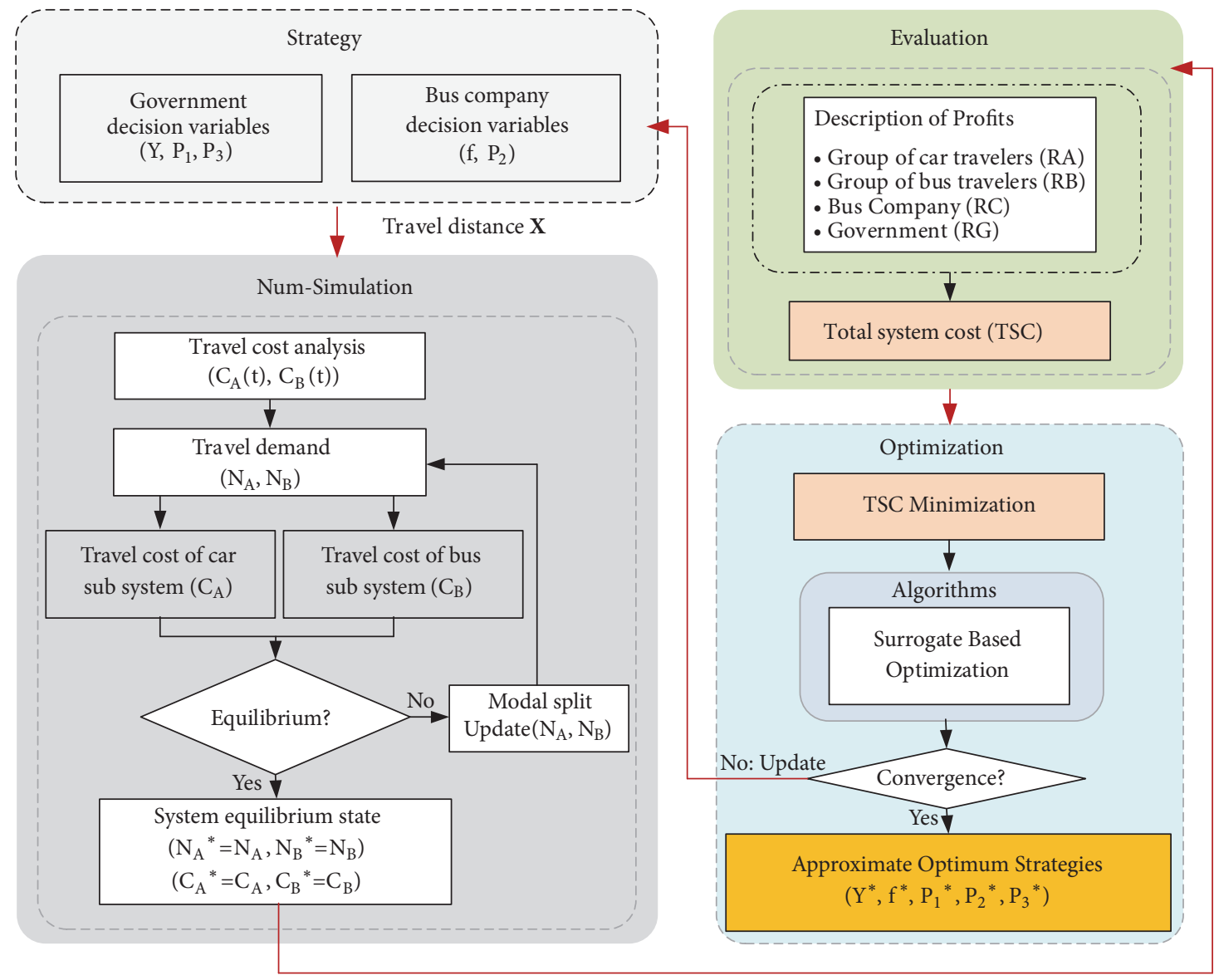

FIGURE 1: Framework for policy-combination oriented optimization.

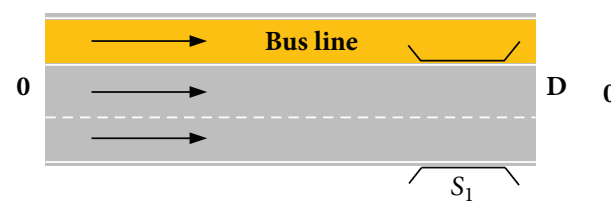

(a) BL scenario

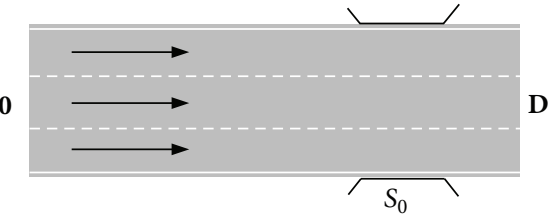

(b) NBL scenario

FIgURe 2: Modelling scenarios.

on kriging surrogate model. The final part is for conclusions and next-step research plans. A framework for policycombination oriented optimization is presented in Figure 1.

\section{Modelling Scenarios and Variables}

In this paper, there are two scenarios, which are presented in Figure 2.

Bus Lane (BL) Scenario: assume the system contains 3 lanes including one bus lane; cars and buses are running on their own lanes and travelers by car are spatially independent from travelers by bus.

None Bus Lane (NBL) Scenario: assume the system contains 3 lanes with no bus lane; cars and buses can use every lane so travelers by car and by bus have interaction with each other.
Before modelling, this paper contains the following assumptions:

(1) Assumption 1: travel demand in peak hours is set as the input of the system and is defined as $N$; the total travel distance is $L \mathrm{~km}$; only two travel modes, car (defined as mode A) and bus (defined as mode B), are taken into account and their corresponding demands are $N_{A}$ and $N_{B}$, respectively; $N=N_{A}+N_{B}$.

(2) Assumption 2: in the BL scenario, the capacity of the bottleneck is defined as $S_{1}$; because of the bus lane priority, cars would queue at the bottleneck but buses do not need to.

(3) Assumption 3: in the NBL scenario, the capacity of the bottleneck is defined as $S_{0}$; both cars and buses need to queue up. 
TABLE 1: Main parameters.

\begin{tabular}{|c|c|c|}
\hline Symbols & Descriptions & Values \\
\hline \multicolumn{3}{|c|}{ Environment Variables } \\
\hline$X$ & Travel distance, $(\mathrm{km})$ & \\
\hline Y & $Y=1:$ BL Scenario; $Y=0$ : NBL Scenario & \\
\hline \multicolumn{3}{|c|}{ Design Variables } \\
\hline$f$ & Bus operating frequency, (bus/h); & $1 \leq f \leq 100$ \\
\hline$P_{1}$ & Travel cost by car, (RMB); & $0 \leq P_{1} \leq 10$ \\
\hline$P_{2}$ & Bus fare, $(\mathrm{RMB})$ & $0 \leq P_{2} \leq 5$ \\
\hline$P_{3}$ & Subsidy for bus travel, (RMB); & $0 \leq P_{3} \leq 5$ \\
\hline \multicolumn{3}{|c|}{ Fixed Parameters } \\
\hline$\alpha_{1}$ & Travelers' time value coefficient; & $\alpha_{1}=20$ \\
\hline$\alpha_{2}$ & Walking time value coefficient; & $\alpha_{2}=30$ \\
\hline$\alpha_{3}$ & Waiting time value coefficient; & $\alpha_{3}=30$ \\
\hline$\alpha_{4}, \alpha_{5}$ & Parameters of time value when staying in the crowded vehicle; & $\alpha_{4}=0.55, \alpha_{5}=-0.074$ \\
\hline$S_{1}$ & Bottleneck capacity in BL Scenario, $(\mathrm{pcu} / \mathrm{h})$ & $S_{1}=1600$ \\
\hline$S_{0}$ & Bottleneck capacity in NBL Scenario, $(\mathrm{pcu} / \mathrm{h})$ & $S_{0}=2400$ \\
\hline$\beta, \gamma$ & Penalty coefficients for arriving earlier or later, $\gamma>\alpha_{1}>\beta$; & $\beta=15, \gamma=30$ \\
\hline$V_{a}, V_{b}$ & Free flow speed of car and bus, respectively, $(\mathrm{km} / \mathrm{h})$; & $V_{a}=30, V_{b}=20$ \\
\hline$T_{1}$ & Car travelers' walking time, $(\mathrm{h})$; & $T_{1}=0.1$ \\
\hline$T_{2}$ & Bus travelers' walking time, $(\mathrm{h})$; & $T_{2}=0.2$ \\
\hline$\delta$ & Price of fuel consumption and car depreciation per $\mathrm{km},(\mathrm{RMB} / \mathrm{km})$; & $\delta=0.88$ \\
\hline$l$ & Distance of every road segment, $(\mathrm{km})$ & $l=1$ \\
\hline$\lambda$ & Passenger car equivalent factor of bus; & $\lambda=3$ \\
\hline$C_{\max }$ & Rated passenger capacity of bus, (person); & $C_{\max }=35$ \\
\hline$N$ & Total travel demand in the system, (person); & $N=5000$ \\
\hline$t^{*}$ & Scheduled arriving time at work; & 9:00 a.m \\
\hline$\theta_{1}$ & Unit variable cost of bus passengers, (RMB/person); & $\theta_{1}=0.1$ \\
\hline$\theta_{2}$ & Unit variable cost of number of buses, $(\mathrm{RMB} / \mathrm{car} * \mathrm{~km})$; & $\theta_{2}=7.0$ \\
\hline
\end{tabular}

(4) Assumption 4: the system follows the principle of first in first out (FIFO).

Table 1 summarizes the main parameters and variables used in the paper.

\section{Travel Cost Analysis}

3.1. Travel Cost of Car (BL Scenario). The car travel cost basically consists of travel time cost $C_{a 1}$, schedule delay cost $C_{a 2}$ generated from arriving to destination earlier or later than required, walking time cost of both ends (from origin to car park and from car park to the destination) $C_{a 3}$, cost of fuel consumption and car depreciation $C_{a 4}$, charges caused from every car trip (parking fee, road pricing fee, etc.) $P_{1}$, and the like.

In BL scenario, when the demand of car travel exceeds the capacity of the bottleneck, cars would queue up. So the travel time for cars contains two parts which are the free flow travel time and waiting time at the bottleneck. Let $t$ be the departure time and let $Q_{1}(t)$ be the queue length at time $t$, then the car travel time cost $C_{a 1}$ can be represented as follows:

$$
C_{a 1}=\alpha_{1}\left[\frac{X l}{V_{a}}+\frac{Q_{1}(t)}{S_{1}}\right]
$$

Schedule delay cost $C_{a 2}$ is

$$
C_{a 2}= \begin{cases}\beta\left[t^{*}-\left(t+\frac{X l}{V_{a}}+\frac{Q_{1}(t)}{S_{1}}\right)\right], & t \in\left[t_{1}, t_{a 1}\right] \\ \gamma\left[\left(t+\frac{X l}{V_{a}}+\frac{Q_{1}(t)}{S_{1}}\right)-t^{*}\right], & t \in\left[t_{a 1}, t_{2}\right]\end{cases}
$$

Walking time cost of both ends is

$$
C_{a 3}=\alpha_{2} T_{1}
$$

Cost of fuel consumption and car depreciation is

$$
C_{a 4}=\delta X l
$$

Hence, in BL scenario, car travel cost function is explained as 


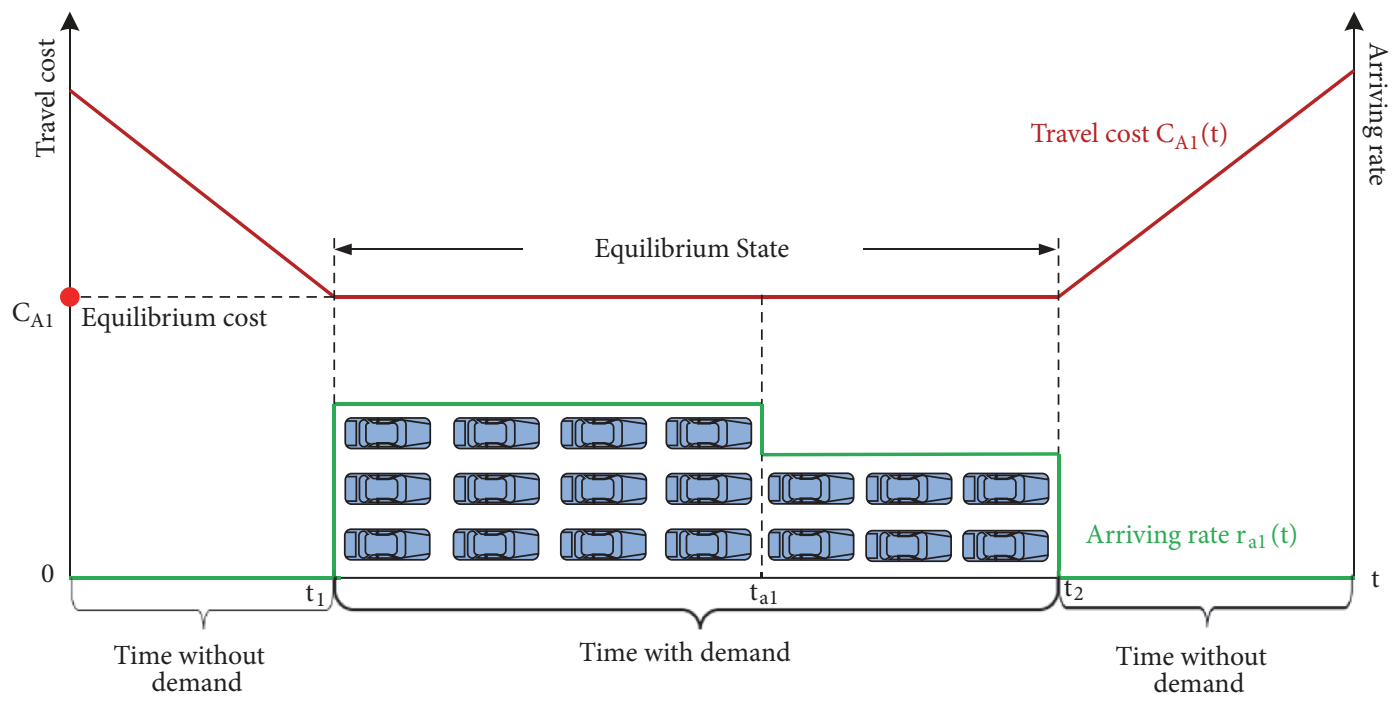

FIgURE 3: Car travel subsystem equilibrium state (UE).

$$
\begin{aligned}
C_{A 1}(t) & =C_{a 1}+C_{a 2}+C_{a 3}+C_{a 4}+P_{1} \\
& =\left\{\begin{array}{l}
\alpha_{1}\left[\frac{X l}{V_{a}}+\frac{Q_{1}(t)}{S_{1}}\right]+\beta\left[t^{*}-\left(t+\frac{X l}{V_{a}}+\frac{Q_{1}(t)}{S_{1}}\right)\right]+\alpha_{2} T_{1}+\delta X l+P_{1}, \quad t \in\left[t_{1}, t_{a 1}\right] \\
\alpha_{1}\left[\frac{X l}{V_{a}}+\frac{Q_{1}(t)}{S_{1}}\right]+\gamma\left[\left(t+\frac{X l}{V_{a}}+\frac{Q_{1}(t)}{S_{1}}\right)-t^{*}\right]+\alpha_{2} T_{1}+\delta X l+P_{1}, \quad t \in\left[t_{a 1}, t_{2}\right]
\end{array}\right.
\end{aligned}
$$

Note that $P_{1}$ is the cost generated during every single car travel including parking fee and road charging; $t_{1}$ and $t_{2}$ are the earliest and the latest points of departure time in the $\mathrm{BL}$ scenario; $t_{a 1}$ is the departure time that car traveler can reach his destination on time, and $t_{a 1}+X l / V_{a}+Q_{1}(t) / S_{1}=t^{*}$.

In the car travel subsystem, each individual has a decision of departure time. In doing so, each faces a trade-off between travel time cost and schedule delay cost. This is a natural extension of the familiar Wardrop user equilibrium (UE) [17]. Equilibrium is obtained when no individual can decrease his travel costs $C_{A 1}(t)$ by altering his departure time $t$, taking all other drivers' departure times as given. Those arriving very early or late experience substantial schedule delay cost but little congestion; those arriving on time experience maximum congestion. Since individuals are identical, the equilibrium is a pure-strategy Nash equilibrium with departure times as the game decisions. This implies that travel cost must be the same at which departures occur [18]. The car travel subsystem equilibrium state can be showed in Figure 3.

Assume that $C_{A 1}$ is the car travel subsystem equilibrium cost in the BL scenario; the departure time choice equilibrium condition is

$$
\begin{aligned}
& C_{A 1}(t)=C_{A 1}, \quad \text { if } r_{a 1}(t)>0\left(t \in\left[t_{1}, t_{2}\right]\right) \\
& C_{A 1}(t)>C_{A 1}, \quad \text { if } r_{a 1}(t)=0\left(t \notin\left[t_{1}, t_{2}\right]\right)
\end{aligned}
$$

For the car travel subsystem equilibrium cost $C_{A 1}$ is constant; its derivation with respect to time must be zero:

$$
\frac{d C_{A 1}(t)}{d t}=\frac{d C_{A 1}}{d t}=0
$$

Suppose the car arriving rate $r_{a 1}(t)$ at the bottleneck is continuous, then the length of the queue can be described as $Q_{1}(t)=\int_{t_{1}}^{t} r_{a 1}(t) d t-S_{1}\left(t-t_{1}\right)$; combined with (9), the car arriving rate is

$$
r_{a 1}(t)= \begin{cases}\frac{\alpha_{1} S_{1}}{\alpha_{1}-\beta}, & t \in\left[t_{1}, t_{a 1}\right] \\ \frac{\alpha_{1} S_{1}}{\alpha_{1}+\gamma}, & t \in\left[t_{a 1}, t_{2}\right]\end{cases}
$$

There are three constraints when the car travel system reaches its equilibrium:

$$
\begin{aligned}
t_{a 1}+\frac{X l}{V_{a}}+\frac{Q_{1}\left(t_{a 1}\right)}{S_{1}} & =t^{*} \\
Q_{1}\left(t_{1}\right) & =Q_{1}\left(t_{2}\right)=0 \\
\int_{t_{1}}^{t_{2}} r_{a 1}(t) d t & =N_{A}
\end{aligned}
$$

Note that (11) expresses the situation that when car traveler takes off at time point $t_{a 1}$, he can get to the destination 
on time. Equation (12) indicates that if setting out at $t_{1}$ or $t_{2}$, traveler will not be confronted with queues while (13) shows that the total demand between these two time points is $N_{A}$. Taken (10) into consideration, it can be obtained that, in the BL scenario, the specific departure time leading to the system equilibrium can be explained as

$$
\begin{aligned}
& t_{1}=t^{*}-\frac{\gamma N_{A}}{(\beta+\gamma) S_{1}}-\frac{X l}{V_{a}} \\
& t_{2}=t^{*}+\frac{\beta N_{A}}{(\beta+\gamma) S_{1}}-\frac{X l}{V_{a}} \\
& t_{a 1}=t^{*}-\frac{\beta \gamma N_{A}}{\alpha_{1}(\beta+\gamma) S_{1}}-\frac{X l}{V_{a}}
\end{aligned}
$$

To sum up, in the BL scenario, the car travel subsystem equilibrium cost (put $t_{1}$ into $C_{A 1}(t)$ ) is

$$
C_{A 1}=\frac{\alpha_{1} X l}{V_{a}}+\frac{\delta N_{A}}{S_{1}}+\alpha_{2} T_{1}+\delta X l+P_{1}
$$

3.2. Travel Cost of Bus (BL scenario). When choosing to travel by bus, the majority of traveler's cost is comprised of bus travel time $C_{b 1}$, cost of the time spent in the crowded vehicle $C_{b 2}$, schedule delay cost $C_{b 3}$ generated from arriving to destination earlier or later than required, cost of time waiting at the bus stop $C_{b 4}$, walking time cost of both ends (from origin to bus stop and from bus stop to the destination) $C_{b 5}$, bus fare $P_{2}$, and so forth.

In the BL scenario, buses and cars run on their own lanes separately. Due to the bus lane priority, buses pass through the bottleneck with no delays. Its travel time can be calculated with free flow speed, so $C_{b 1}$ is expressed as

$$
\begin{gathered}
C_{b 1}=\frac{\alpha_{1} X l}{V_{b}} \\
C_{B 1}\left(t_{j_{1}}\right)=C_{b 1}+C_{b 2}+C_{b 3}+C_{b 4}+C_{b 5}+P_{2}
\end{gathered}
$$$$
=\left\{\begin{array}{l}
{\left[\alpha_{1}+g\left(n_{j}\right)\right] \frac{X l}{V_{b}}+\beta\left[t^{*}-\left(t_{j_{1}}+\frac{X l}{V_{b}}\right)\right]+\alpha_{2} T_{2}+\frac{\alpha_{3}}{2 f}+P_{2}, \quad t_{j_{1}} \in\left[t_{e_{1}}, t_{b 1}\right]} \\
{\left[\alpha_{1}+g\left(n_{j}\right)\right] \frac{X l}{V_{b}}+\gamma\left[\left(t_{j_{1}}+\frac{X l}{V_{b}}\right)-t^{*}\right]+\alpha_{2} T_{2}+\frac{\alpha_{3}}{2 f}+P_{2}, \quad t_{j_{1}} \in\left[t_{b 1}, t_{-h_{1}}\right]}
\end{array}\right.
$$

where $t_{b 1}$ is the departure time that bus traveler can get to the destination on time. When $j=0$, then $t_{b 1}+X l / V_{b}=t^{*} ; t_{e_{1}}$, $t_{-h_{1}}$ are the earliest and latest departure time for bus travelers in the BL scenario.
The cost of time spent in the crowded bus equals the product of its time value and bus travel time, the cost function of which $C_{b 2}$ is

$$
C_{b 2}=\frac{g\left(n_{j}\right) X l}{V_{b}}
$$

where $j$ stands for the order of buses; $n_{j}$ is number of passengers in the $j$ th bus. Taking the passengers' in-vehicle comfort into consideration [19], the time value function of the time spent in the crowded bus $g\left(n_{j}\right)$ can be built as

$$
\begin{aligned}
& g\left(n_{j}\right) \\
& = \begin{cases}0 & \left(0 \leq \frac{n_{j}}{C_{\max }} \leq 0.8\right) \\
\alpha_{1} \alpha_{4}\left(\frac{n_{j}}{C_{\max }}-0.8\right) e^{-\alpha_{5}\left(n_{j} / C_{\max }-0.8\right)^{2}} & \left(\frac{n_{j}}{C_{\max }}>0.8\right)\end{cases}
\end{aligned}
$$

Schedule delay cost for unpunctuality $C_{b 3}$ is

$$
C_{a 3}= \begin{cases}\beta\left[t^{*}-\left(t_{j_{1}}+\frac{X l}{V_{b}}\right)\right], & t_{j_{1}} \in\left[t_{e_{1}}, t_{b 1}\right] \\ \gamma\left[\left(t_{j_{1}}+\frac{X l}{V_{b}}\right)-t^{*}\right], & t_{j_{1}} \in\left[t_{b 1}, t_{-h_{1}}\right]\end{cases}
$$

where $t_{j_{1}}$ represents the departure time of the $j$ th bus in the BL scenario; let $j=0$ when bus can arrive destination on time, then all the buses' departure time points can be stated as

$$
t_{j_{1}}=t^{*}-\frac{X l}{V_{b}}-\frac{j}{f}
$$

Cost of waiting time at the bus stop $C_{b 4}$ is

$$
C_{b 4}=\frac{\alpha_{3}}{2 f}
$$

Cost of walking time $C_{b 5}$ is

$$
C_{b 5}=\alpha_{2} T_{2}
$$

Hence, in the BL scenario, bus travel cost function is
In the $\mathrm{BL}$ scenario, bus travelers make game decision between arrival time loss penalty and the comfort level in the bus by choosing to take different shift number of bus to finally reach the lowest travel cost. The subsystem reaches its 
equilibrium when no traveler in it can lower his travel cost $C_{B 1}\left(t_{j_{1}}\right)$ by choosing different shift of bus. Assume that $C_{B 1}$ is the bus travel subsystem equilibrium cost in the BL scenario; then the condition of the bus travel subsystem equilibrium is

$$
\begin{array}{ll}
C_{B 1}\left(t_{j_{1}}\right)=C_{B 1} & \text { if } n_{j}>0 \\
C_{B 1}\left(t_{j_{1}}\right)>C_{B 1} & \text { if } n_{j}=0
\end{array}
$$

where $\sum_{j \in Z} n_{j}=N_{B}$.
3.3. Travel Cost of Car (NBL Scenario). Different from BL scenario, none of the cars and buses have priority on road in the NBL scenario as all vehicles need to wait in line to pass the bottleneck. Despite this, the composition of car travel cost is similar to that in BL scenario including travel time cost, schedule delay cost generated from arriving to destination earlier or later than required, walking time cost of both ends (from origin to car park and from car park to the destination), cost of fuel consumption and car depreciation, and charges caused from every car trip. The travel cost function is

$$
\begin{aligned}
C_{A 0}(t) & =C_{a 1}^{\prime}+C_{a 2}^{\prime}+C_{a 3}+C_{a 4}+P_{1} \\
& = \begin{cases}\alpha_{1}\left[\frac{X l}{V_{a}}+\frac{Q_{0}(t)}{S_{0}}\right]+\beta\left[t^{*}-\left(t+\frac{X l}{V_{a}}+\frac{Q_{0}(t)}{S_{0}}\right)\right]+\alpha_{2} T_{1}+\delta X l+P_{1}, & t \in\left[t_{5}, t_{a 0}\right] \\
\alpha_{1}\left[\frac{X l}{V_{a}}+\frac{Q_{0}(t)}{S_{0}}\right]+\gamma\left[\left(t+\frac{X l}{V_{a}}+\frac{Q_{0}(t)}{S_{0}}\right)-t^{*}\right]+\alpha_{2} T_{1}+\delta X l+P_{1}, & t \in\left[t_{a 0}, t_{6}\right]\end{cases}
\end{aligned}
$$

where $Q_{0}(t)$ is the queue length when leaving at time $t ; t_{5}$ and $t_{6}$ are the earliest and latest departure time in the NBL scenario; $t_{a 0}$ is the perfect departure time that car traveler can get to the destination on time, and $t_{a 0}+X l / V_{a}+Q_{0}(t) / S_{0}=t^{*}$.

Travel behavior of car travelers is similar in either BL or NBL scenario when the subsystem reaches its equilibrium, so the subsystem equilibrium cost is

$$
C_{A 0}=\frac{\alpha_{1} X l}{V_{a}}+\frac{\beta \gamma}{\beta+\gamma}\left(\frac{N_{A}}{S_{0}-\lambda f}\right)+\alpha_{2} T_{1}+\delta X l+P_{1}
$$

The corresponding departure time of the subsystem equilibrium stands for

$$
t_{5}=t^{*}-\frac{\gamma}{\beta+\gamma}\left(\frac{N_{A}}{S_{0}-\lambda f}\right)-\frac{X l}{V_{a}}
$$

$$
\begin{aligned}
C_{B 0}\left(t_{j_{0}}\right) & =C_{b 1}^{\prime}+C_{b 2}^{\prime}+C_{b 3}^{\prime}+C_{b 4}+C_{b 5}+P_{2} \\
& =\left\{\begin{array}{l}
{\left[\alpha_{1}+g\left(n_{j}\right)\right]\left[\frac{X l}{V_{b}}+\frac{Q_{0}\left(t_{j_{0}}\right)}{S_{0}}\right]+\beta\left[t^{*}-\left(t_{j}+\frac{X l}{V_{b}}+\frac{Q_{0}\left(t_{j_{0}}\right)}{S_{0}}\right)\right]+\alpha_{2} T_{2}+\frac{\alpha_{3}}{2 f}+P_{2} \quad t_{j_{0}} \in\left[t_{e_{0}}, t_{b 0}\right]} \\
{\left[\alpha_{1}+g\left(n_{j}\right)\right]\left[\frac{X l}{V_{b}}+\frac{Q_{0}\left(t_{j_{0}}\right)}{S_{0}}\right]+\gamma\left[\left(t_{j}+\frac{X l}{V_{b}}+\frac{Q_{0}\left(t_{j_{0}}\right)}{S_{0}}\right)-t^{*}\right]+\alpha_{2} T_{2}+\frac{\alpha_{3}}{2 f}+P_{2} \quad t_{j_{0}} \in\left[t_{b 0}, t_{-h_{0}}\right]}
\end{array}\right.
\end{aligned}
$$

where $t_{e_{0}}, t_{-h_{0}}$ indicate the earliest and latest departure time in the NBL scenario; $t_{b 0}$ is the specific departure time to reach the destination on time:

$$
\begin{aligned}
t_{b 0}= & \frac{\beta X l}{\alpha_{1}}\left(\frac{1}{V_{b}}-\frac{1}{V_{a}}\right)+t^{*}-\frac{X l}{V_{b}} \\
& -\frac{\beta \gamma}{\alpha_{1}(\beta+\gamma)}\left(\frac{N_{A}}{S_{0}-\lambda f}\right)
\end{aligned}
$$

$$
\begin{aligned}
& t_{6}=t^{*}+\frac{\beta}{\beta+\gamma}\left(\frac{N_{A}}{S_{0}-\lambda f}\right)-\frac{X l}{V_{a}} \\
& t_{a 0}=t^{*}-\frac{\beta \gamma}{\alpha_{1}(\beta+\gamma)}\left(\frac{N_{A}}{S_{0}-\lambda f}\right)-\frac{X l}{V_{a}}
\end{aligned}
$$

3.4. Travel Cost of Bus (NBL Scenario). The majority of the bus travel cost in the NBL scenario consists of bus travel time, cost of the time spent in the crowded vehicle, schedule delay cost generated from arriving to destination earlier or later than required, cost of time waiting at the bus stop, walking time cost of both ends (from origin to bus stop and from bus stop to the destination), bus fare, and so forth. The travel time of the buses contains not only the travel time with free flow speed, but also the waiting time at the bottleneck since the bus has no priority. So in this circumstance, the travel cost should be
Departure time $t_{j_{0}}$ for all the shifts of bus in NBL scenario:

$$
\begin{aligned}
t_{j_{0}}= & \frac{\beta X l}{\alpha_{1}}\left(\frac{1}{V_{b}}-\frac{1}{V_{a}}\right)+t^{*}-\frac{X l}{V_{b}} \\
& -\frac{\beta \gamma}{\alpha_{1}(\beta+\gamma)}\left(\frac{N_{A}}{S_{0}-\lambda f}\right)-\frac{j}{f}
\end{aligned}
$$


In the NBL scenario, bus travelers make decision among arrival time loss penalty, the comfort level in the bus, and the queuing at the bottleneck, by choosing to take different shift number of bus to finally reach the lowest travel cost. Assume that $C_{B 0}$ is the bus travel subsystem equilibrium cost in the NBL scenario, then the condition of the bus travel subsystem equilibrium is

$$
\begin{aligned}
& C_{B 0}\left(t_{j_{0}}\right)=C_{B 0} \text { if } n_{j}>0 \\
& C_{B 0}\left(t_{j_{0}}\right)>C_{B 0} \text { if } n_{j}=0
\end{aligned}
$$

where $\sum_{j \in Z} n_{j}=N_{B}$.

3.5. Overall System Equilibrium. When the car travel subsystem equilibrium $\operatorname{cost} C_{A i}(i=0$ or 1$)$ equals the bus travel subsystem equilibrium cost $C_{B i}$, overall system equilibrium is achieved. That is, under a specific policy $\left(f, P_{1}, P_{2}, P_{3}\right)$ when overall system equilibrium is realized, the equilibrium condition should be satisfied:

$$
C_{A i}=C_{B i}, \quad i=0 \text { or } 1
$$

In order to facilitate the solution of the model, we consider that the overall system reaches an approximate equilibrium when the following conditions are met.

$$
\left|C_{A i}-C_{B i}\right| \leq \varepsilon, \quad i=0 \text { or } 1
$$

where $\varepsilon$ is the system overall equilibrium judgment threshold, which is very small constant.

\section{Policy Optimization Model Based on Stackelberg Game}

4.1. Description of Profits for Game Groups. In the study, travelers, the government, and the bus company are the main three groups in the transport system whose profits are described.

The group of travelers mainly consists of car travelers and bus travelers. The total cost generated by car travelers can be represented as the product of total car travel demand and car travel cost. When the system reaches equilibrium, the cost of all car travelers is

$$
R_{A}=C_{A} N_{A}
$$

Similarly, the total cost generated by bus travelers equals total bus travel demand multiplied by bus travel cost. When the system reaches equilibrium, the cost of all bus travelers is

$$
R_{B}=C_{B} N_{B}
$$

Therefore, the total cost of travelers in the system is

$$
R_{T}=C_{A} N_{A}+C_{B} N_{B}
$$

The profit earned by the bus company mainly includes bus fare and the subsidy offered by the government. Taking the payment for operation into consideration, the total cost of the bus company is

$$
R_{C}=\left(\theta_{1} N_{B}+\theta_{2} F\right)-P_{2} N_{B}-P_{3} N_{B}
$$

The income of the government primarily comes from car travelers with their expense of car parking, road pricing fee, and so forth. In the meantime, the government is also in charge of bus subsidy and road maintenance. Hence, the cost of the government is

$$
R_{G}=P_{3} N_{B}-P_{1} N_{A}+B
$$

In summary, the total system cost (TSC) is expressed as

$$
T S C=R_{T}+R_{C}+R_{G}
$$

4.2. Game Model. Urban public transport system is a macrosystem which is both open and complicated. There is a massive number of transport participants with their own aims and preference that will influence each other by the decision among travelers or between policy makers and travelers (Leader-Follower). For the latter, policy makers adjust the control strategy to guide the travelers to change their travel plan. Once travelers react to the policy implementation, the system evolves with it to pass to its state as the feedback to the policy makers so that they can again make some improvements. This circulation stops when it reaches the equilibrium and system optimal.

This paper pays attention to the dynamic adjustment between transport participants in the system and studies on the dynamic interactions between policy makers, the bus company, and travelers by establishing the Stackelberg game model. The model structure can be shown as Figure 4. Here, Stackelberg model is a bilevel game model whose upper level stands for traffic policy maker (Leader) and the other is traveler (Follower). On one hand, Leader takes minimizing TSC as the goal and carries out policy combination $\left(f, P_{1}, P_{2}, P_{3}\right)$ by predicting travelers' behavior, while on the other hand, travelers change their departure time and travel mode to reduce the travel cost under the new policy as a procedure of Nash game to finally get to the new system equilibrium $\left(N_{A}^{*}, N_{B}^{*}, C_{A}^{*}, C_{B}^{*}\right)$.

Below is the Stackelberg model built in this research:

\section{Upper Level}

$$
\begin{aligned}
& \min \operatorname{TSC}\left(\left(f, P_{1}, P_{2}, P_{3}\right) \mid\left(N_{A}^{*}, N_{B}^{*}, C_{A}^{*}, C_{B}^{*}\right)\right) \\
& \quad=R_{T}+R_{C}+R_{G}
\end{aligned}
$$

Lower Level

$$
\left(N_{A}^{*}, N_{B}^{*}, C_{A}^{*}, C_{B}^{*}\right) \in \arg \min \left|C_{A}-C_{B}\right| \leq \varepsilon
$$




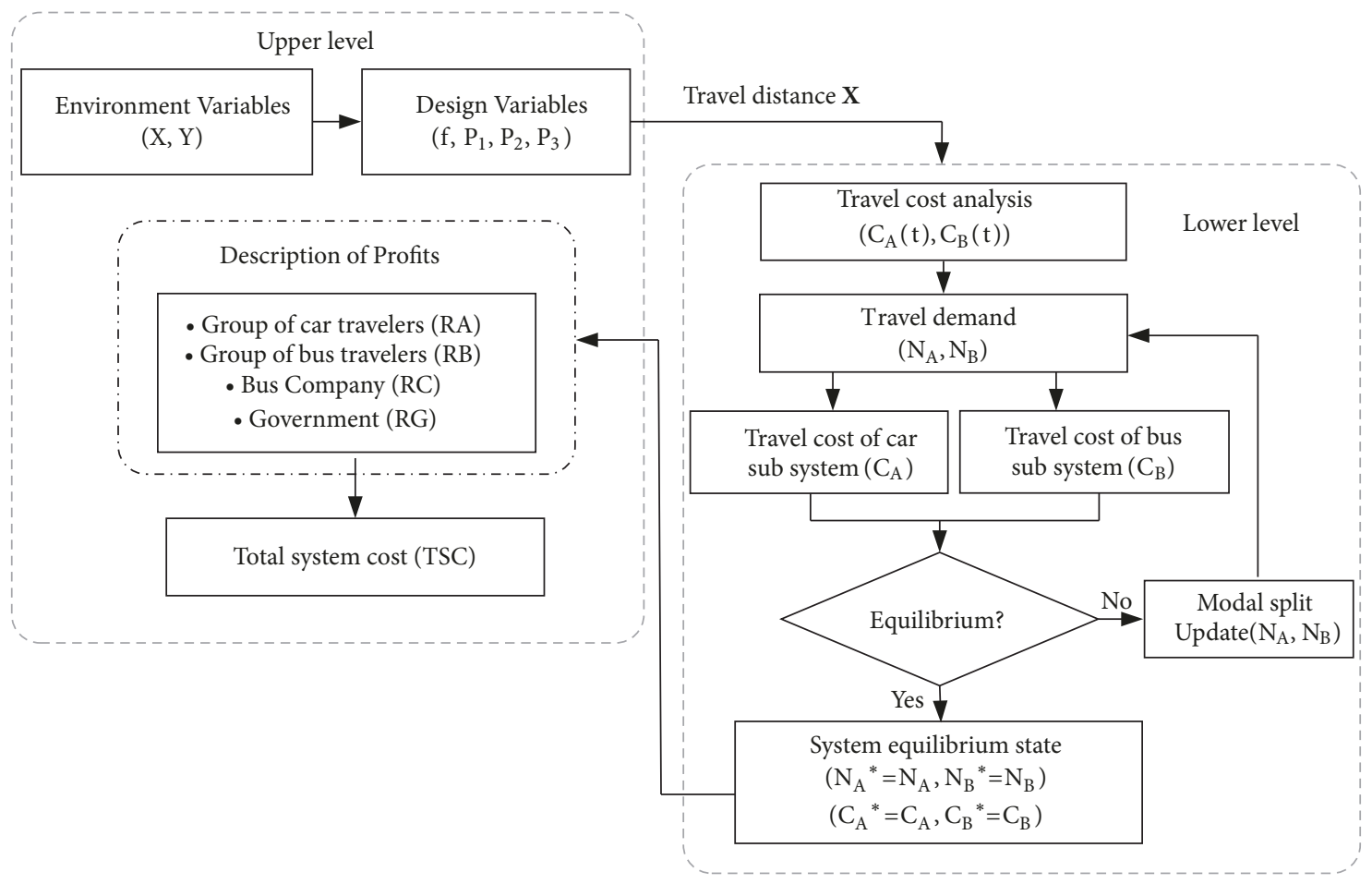

FIGURE 4: Conceptual framework of the numerical simulation system.

subject to

$$
\begin{aligned}
N_{A}+N_{B} & =N \\
1 & \leq f \leq 100 \\
0 & \leq P_{1} \leq 10 \\
0 & \leq P_{2} \leq 5 \\
0 & \leq P_{3} \leq 5
\end{aligned}
$$

where $\left(N_{A}^{*}, N_{B}^{*}, C_{A}^{*}, C_{B}^{*}\right)$ is the numbers of car and bus travelers and travelers' cost under a certain policy combination when the system reaches equilibrium.

\section{Surrogate Based Optimization}

5.1. Methodology. The optimization algorithm of surrogate based model $[20,21]$ is adopted as an approximate global optimization algorithm in the study. The basic idea is to describe the relationship between objective function of optimization and design variables as the approximate global optimization algorithm. The iteration process is achieved using approximate response surface model in place of the source function to evaluate. This algorithm can effectively deal with massive multidimension optimization model, optimization problem of simulation model with no direct analytic expressions, and so forth. The steps of creating the surrogate based model in the paper are as follows:

(1) Initial Samples. Latin square design (LHS) [22] is adopted to determine the locations of sampling points $\mathbf{x}^{(i)}$ for the model. Note that $\mathbf{x}^{(i)}=\left(f, P_{1}, P_{2}, P_{3}\right)(i=$ $(1, \cdots n))$ is a four-dimensioned point.

(2) Num-Simulation. It is to form a group of sample pairs of $\mathbf{y}^{(i)}$ responding from sampling points $\mathbf{x}^{(i)}$ acquired by the numerical simulation model built in the paper.

(3) Response Surface Construction. This step is to construct a response surface using kriging surrogate model. To find new policy combinations based on the initial samples, we consider two-stage model by infilling points to the initial set using criteria such as the expectation of improvement (EI) across the response surface.

(4) Model Validation. It is to assess and validate the assumed surrogate model by comparing an additional test set of objective function data with values estimated by the surrogates at points corresponding to the variables at which the independent objective function values are calculated. If the accuracy is accepted, then stop testing; otherwise, continue to update the model until the accuracy is accepted.

Finally, we find the optimal solution of the policy combination using the optimized surrogate models. In this article, we apply genetic algorithm (GA) to seek the global optima for the estimated response surface. The optimization algorithm of surrogate based model flow chart is displayed in Figure 5.

5.2. Initial Samples. In this part, initial samples in both BL and NBL scenario are created based on LHS method. Initial training set is built by the responding value of every sample 


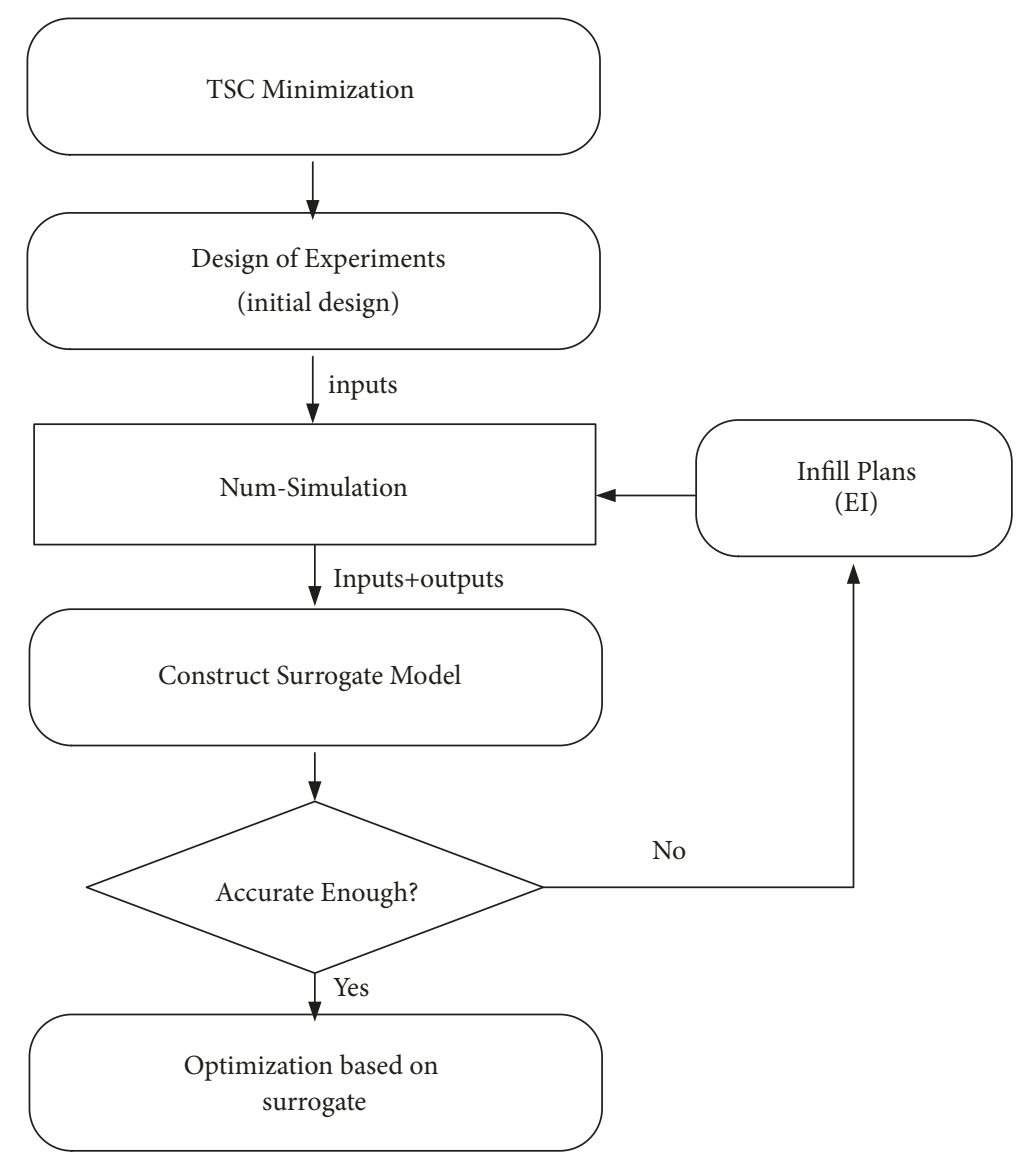

FIGURE 5: Num-simulation optimization procedure using the surrogate methodology.

calculated from the numerical simulation system. Table 2 shows the initial training set based on both scenarios.

5.3. Surrogate Model. In this paper, we construct a response surface using kriging surrogate model. The kriging response surface $[23,24]$ is formed by two parts, one of which is the regression of the data for the process average while the other is the stochastic process model established by data observed and its quantified correlation. The model is expressed as

$$
\widehat{y}_{r}=\widehat{\mu}_{r}+\psi^{T}(\psi+\lambda \mathbf{I})^{-1}\left(y-1 \widehat{\mu}_{r}\right)
$$

where $\widehat{\mu}_{r}$ is the process average which is calculated by

$$
\widehat{\mu}_{r}=\frac{\mathbf{1}^{\mathbf{T}}(\psi+\lambda \mathbf{I})^{-1} \mathbf{y}}{\mathbf{1}^{\mathrm{T}}(\psi+\lambda \mathbf{I})^{-1} \mathbf{1}}
$$

$\psi^{(i)}$ is the base function:

$$
\psi^{(i)}=\exp \left(-\sum_{j=1}^{k} \theta_{j}\left|x_{j}^{(i)}-x_{j}\right|^{p_{j}}\right)
$$

$\operatorname{cor}\left[Y\left(x^{(i)}\right), Y\left(x^{(l)}\right)\right]$ is correlation of random variables which is expressed as

$$
\operatorname{cor}\left[Y\left(x^{(i)}\right), Y\left(x^{(l)}\right)\right]=\exp \left(-\sum_{j=1}^{k} \theta_{j}\left|x_{j}^{(i)}-x_{j}^{(l)}\right|^{p_{j}}\right)
$$

$\psi$ is a correlation matrix $(n \times n)$ :

$$
=\left(\begin{array}{ccc}
\operatorname{cor}\left[Y\left(x^{(1)}\right), Y\left(x^{(1)}\right)\right] & \cdots & \operatorname{cor}\left[Y\left(x^{(1)}\right), Y\left(x^{(n)}\right)\right] \\
\vdots & \ddots & \vdots \\
\operatorname{cor}\left[Y\left(x^{(n)}\right), Y\left(x^{(1)}\right)\right] & \cdots & \operatorname{cor}\left[Y\left(x^{(n)}\right), Y\left(x^{(n)}\right)\right]
\end{array}\right)
$$

After training the model, the accuracy of it needs to be improved to meet the needs. To achieve this, the estimated standard deviation is used together with the infill criterion based on the maximized expected increment max (EI) [25] to gradually increase the sample size and improve the global estimation and the optimization accuracy until it is accepted. The expression of data encryption is

$$
\begin{aligned}
& E I(x)=\frac{1}{\sqrt{2 \pi} \widehat{s}(x)} \\
& \quad \cdot \int_{-\infty}^{y_{\min }}\left(y_{\min }-u\right) \exp \left(\frac{(u-\widehat{f}(x))^{2}}{2 \widehat{s}(x)^{2}}\right) d u
\end{aligned}
$$

where $E I(x)$ is the EI value of data $\mathrm{x} ; y_{\min }$ is the minimal within all the training set. 
TABLE 2: Initial training sets based on LHS ( $\mathrm{L}=20 \mathrm{Km})$.

\begin{tabular}{|c|c|c|c|c|c|c|}
\hline Sample & $\mathrm{f}$ & P1 & $\mathrm{P} 2$ & P3 & TSC(BL) & TSC(NBL) \\
\hline 1 & 60.40 & 2.00 & 1.67 & 5.00 & 185777.91 & 225677.89 \\
\hline 2 & 93.40 & 3.33 & 3.00 & 3.67 & 186796.77 & 204368.78 \\
\hline 3 & 80.20 & 4.00 & 4.33 & 0.33 & 185024.12 & 213703.29 \\
\hline 4 & 86.80 & 10.00 & 2.67 & 1.67 & 208164.83 & 204523.45 \\
\hline 5 & 7.60 & 4.67 & 2.33 & 0.00 & 184429.85 & 267614.63 \\
\hline 6 & 67.00 & 1.33 & 0.00 & 1.33 & 189456.57 & 219378.16 \\
\hline 7 & 1.00 & 5.33 & 4.00 & 3.33 & 183540.38 & 273631.74 \\
\hline 8 & 100.00 & 7.33 & 0.33 & 2.33 & 202561.09 & 198485.98 \\
\hline 9 & 47.20 & 0.67 & 2.00 & 2.00 & 182491.73 & 238477.61 \\
\hline 10 & 27.40 & 8.67 & 3.33 & 2.67 & 194053.19 & 240229.91 \\
\hline 11 & 40.60 & 8.00 & 1.33 & 0.67 & 204952.75 & 230611.79 \\
\hline 12 & 34.00 & 6.00 & 5.00 & 1.00 & 185727.60 & 245151.34 \\
\hline 13 & 53.80 & 9.33 & 1.00 & 4.67 & 220477.97 & 225774.28 \\
\hline 14 & 14.20 & 2.67 & 0.67 & 3.00 & 185137.01 & 256741.38 \\
\hline 15 & 73.60 & 6.67 & 3.67 & 4.33 & 196209.59 & 208643.40 \\
\hline 16 & 20.80 & 0.00 & 4.67 & 4.00 & 182221.75 & 261805.43 \\
\hline lower & 1.00 & 0.00 & 0.00 & 0.00 & 183539.01 & 273797.40 \\
\hline upper & 100.00 & 10.00 & 5.00 & 5.00 & 202561.09 & 198485.98 \\
\hline baseline & 60.00 & 5.00 & 2.00 & 2.00 & 194109.49 & 219819.26 \\
\hline
\end{tabular}

5.4. Evaluation Criteria. There are 6 indexes adopted to evaluate the accuracy of the surrogate model in the paper.

(1) Root mean square error (RMSE) indicates the global error:

$$
R M S E=\sqrt{\frac{1}{\mathrm{n}} \sum_{i=1}^{n}\left(f\left(\mathbf{x}^{(i)}\right)-\widehat{f}\left(\mathbf{x}^{(i)}\right)\right)^{2}}
$$

(2) Maximum absolute error (MAE) indicates local error:

$$
M A E=\max _{1 \leq i \leq n}\left|f\left(\mathbf{x}^{(i)}\right)-\widehat{f}\left(\mathbf{x}^{(i)}\right)\right|
$$

(3) Normalized root mean square error (NRMSE) is

$$
N R M S E=\sqrt{\frac{\sum_{i=1}^{n}\left[f\left(\mathbf{x}^{(i)}\right)-\widehat{f}\left(\mathbf{x}^{(i)}\right)\right]^{2}}{\sum_{i=1}^{n}\left(f\left(\mathbf{x}^{(i)}\right)\right)^{2}}}
$$

(4) Normalized maximum absolute error (NMAE) is

$$
N M A E=\frac{\max _{1 \leq i \leq n}\left|f\left(\mathbf{x}^{(i)}\right)-\widehat{f}\left(\mathbf{x}^{(i)}\right)\right|}{\sqrt{(1 / \mathrm{n}) \sum_{i=1}^{n}\left(f\left(\mathbf{x}^{(i)}\right)-\bar{f}\right)^{2}}}
$$

where $\bar{f}=(1 / \mathrm{n}) \sum_{i=1}^{n} \widehat{f}\left(\mathbf{x}^{(i)}\right)$.

(5) Estimated global optimal (EGO) is

$$
E G O=\widehat{f}\left(\widehat{\mathbf{x}}^{*}\right)
$$

where $\mathbf{x}^{*}=\arg \min f(\mathbf{x}), \widehat{\mathbf{x}}^{*}=\arg \min \widehat{f}(\mathbf{x})$.
(6) Pearson correlation coefficient (PCC) (the more the index is approaching value 1 , the more accurate the model will be; index value equals 0 means uncorrelated) is

$$
=\left(\frac{N \sum \hat{f} f-\sum f \sum \hat{f}}{\sqrt{\left[N \sum f^{2}-\left(\sum f\right)^{2}\right]\left[N \sum \hat{f}^{2}-\left(\sum \hat{f}\right)^{2}\right]}}\right)^{2}
$$

5.5. Results. In this paper, the initial surrogate model is established using initial training sample and continuously updated on account of infill criteria. At the end of 22nd and 129th update in two scenarios, the accuracy of the model is accepted and the corresponding policy combination is output along with its accuracy indexes. The optimization results and relevant indexes are shown in Tables 3 and 4 and the system equilibrium is revealed in Table 5 .

The point of $x_{\text {baseline }}=(60,5.00,2.00,2.00)$ is selected as the input for initial policy (base point). This indicates the initial set that the bus departure frequency $f=60$ (bus/h), the cost generated from the car travel (parking fee, road pricing fee, etc.) $P_{1}=5.00(\mathrm{RMB})$, bus fare $P_{2}=2.00$ (RMB), and bus fare subsidy for one trip $P_{3}=2.00$ (RMB). The total system costs are $T S C=194109.49(\mathrm{RMB})$ and $T S C=219819.26(\mathrm{RMB})$ in BL scenario and NBL scenario, respectively.

In the BL scenario, the calculated optimal solution $x_{B L}^{\text {opt }}$ $=(100,0.00,5.00,2.50)$, indicating that the bus departure frequency $f=100$ (bus/h), travel cost generated from car travel (parking, road pricing fee, etc.) $P_{1}=0.00$ (RMB), bus fare $P_{2}$ $=5.00(\mathrm{RMB})$, and bus fare subsidy for a single trip $P_{3}=2.50$ 
TABLE 3: Policy combination output in different scenarios.

\begin{tabular}{lcccccccc}
\hline Scenario & & $\mathrm{f}$ & $\mathrm{P} 1$ & $\mathrm{P} 2$ & P3 & $\begin{array}{c}\text { TSC } \\
\text { Estimated }\end{array}$ & $\begin{array}{c}\text { TSC } \\
\text { Simulated }\end{array}$ & $\begin{array}{c}\text { TSC } \\
\text { Decrease (\%) }\end{array}$ \\
\hline \multirow{2}{*}{ BL } & Optimal & 100 & 0.00 & 5.00 & 2.50 & 177443.72 & 177444.61 & $8.59 \%$ \\
& Baseline & 60 & 5.00 & 2.00 & 2.00 & 194109.63 & 194109.49 & 198222.93 \\
\multirow{2}{*}{ NBL } & Optimal & 100 & 3.34 & 0.94 & 0.00 & 198351.84 & $9.82 \%$ \\
& Baseline & 60 & 5.00 & 2.00 & 2.00 & 219820.16 & 219819.26 \\
\hline
\end{tabular}

TABLE 4: Model accuracy in different scenarios.

\begin{tabular}{lccccc}
\hline Scenario & RMSE & MAE & NRMSE & NMAE & EGO \\
BL & 961.69 & 1702.80 & $0.50 \%$ & 0.21 & 177443.72 \\
NBL & 1333.98 & 3085.53 & $0.57 \%$ & 0.13 & 198351.84 \\
\hline
\end{tabular}

TABLE 5: System equilibrium in different scenarios.

\begin{tabular}{|c|c|c|c|c|c|}
\hline \multicolumn{3}{|c|}{$\mathrm{BL}$} & \multicolumn{3}{|c|}{ NBL } \\
\hline Index & Baseline & Optimal solution & Index & Baseline & Optimal solution \\
\hline $\mathrm{L}$ & 20 & 20 & $\mathrm{~L}$ & 20 & 20 \\
\hline $\mathrm{Y}$ & 1 & 1 & $\mathrm{Y}$ & 0 & 0 \\
\hline $\mathrm{f}$ & 60 & 100 & $\mathrm{f}$ & 60 & 100 \\
\hline $\mathrm{P}_{1}$ & 5.00 & 0.00 & $\mathrm{P}_{1}$ & 5.00 & 3.34 \\
\hline $\mathrm{P}_{2}$ & 2.00 & 5.00 & $\mathrm{P}_{2}$ & 2.00 & 0.94 \\
\hline $\mathrm{P}_{3}$ & 2.00 & 2.50 & $\mathrm{P}_{3}$ & 2.00 & 0.00 \\
\hline $\mathrm{N}_{\mathrm{A}}$ & 1824 & 3401 & $\mathrm{~N}_{\mathrm{A}}$ & 1320 & 87 \\
\hline $\mathrm{N}_{\mathrm{B}}$ & 3176 & 1599 & $\mathrm{~N}_{\mathrm{B}}$ & 3680 & 4913 \\
\hline $\mathrm{C}_{\mathrm{A}}$ & 39.94 & 35.80 & $\mathrm{C}_{\mathrm{A}}$ & 44.88 & 37.69 \\
\hline $\mathrm{t}_{1}$ & 7.57 & 6.92 & $t_{5}$ & 7.94 & 8.31 \\
\hline$t_{2}$ & 8.71 & 9.04 & $t_{6}$ & 8.53 & 8.35 \\
\hline $\mathrm{t}_{\mathrm{al}}$ & 7.76 & 7.27 & $\mathrm{t}_{\mathrm{a} 0}$ & 8.04 & 8.31 \\
\hline $\mathrm{C}_{\mathrm{B}}$ & 39.91 & 35.78 & $\mathrm{C}_{\mathrm{B}}$ & 44.40 & 37.67 \\
\hline$t_{b 1}$ & 8 & 8.00 & $t_{b 0}$ & 7.95 & 8.23 \\
\hline $\mathrm{F}$ & 69 & 45 & $\mathrm{~F}$ & 77 & 102 \\
\hline $\mathrm{R}_{\mathrm{A}}$ & 72849.14 & 121777.10 & $\mathrm{R}_{\mathrm{A}}$ & 59254.99 & 3282.28 \\
\hline $\mathrm{R}_{\mathrm{B}}$ & 126755.12 & 57201.55 & $\mathrm{R}_{\mathrm{B}}$ & 163377.14 & 185068.63 \\
\hline $\mathrm{R}_{\mathrm{C}}$ & -2725.93 & -5531.01 & $\mathrm{R}_{\mathrm{C}}$ & -3570.90 & 10162.98 \\
\hline $\mathrm{R}_{\mathrm{G}}$ & -2768.84 & 3996.96 & $\mathrm{R}_{\mathrm{G}}$ & 758.03 & -290.96 \\
\hline TSC & 194109.49 & 177444.61 & TSC & 219819.26 & 198222.93 \\
\hline
\end{tabular}

(RMB). When the system reaches equilibrium, the bus travel demand $N_{B}$ is equal to 1599 (person) and the corresponding travel cost is 35.78 (RMB); the car travel demand $N_{A}$ is equal to 3401 (person) and the corresponding travel cost is 35.80 (RMB). Hence, the optimal total system cost TSC $=177444.61$ (RMB), showing $8.95 \%$ decrease compared with the base point.

In the NBL scenario, the calculated optimal solution $=(100,3.34,0.94,0.00)$, indicating that the bus departure frequency $f=100$ (bus $/$ h), travel cost generated from car travel (parking, road pricing fee, etc.) $P_{1}=3.34$ (RMB), bus fare $P_{2}$ $=0.94(\mathrm{RMB})$, and bus fare subsidy for a single trip $P_{3}=0.00$ (RMB). When the system reaches equilibrium, the bus travel demand $N_{B}$ is equal to 4913 (person) and the corresponding travel cost is 37.67 (RMB); the car travel demand $N_{A}$ is equal to 87 (person) and the corresponding travel cost is 37.69 (RMB). Hence, the optimal total system cost TSC $=198222.93$ (RMB), showing $9.82 \%$ decrease compared with the base point.

\section{Conclusion}

The paper proposes a model based on the game theory to model the policy and resource allocation in the transport system which provides an idea for policy-combination oriented optimization of urban transportation system in the future. The main contribution of the study can be summed up into four conclusions: (a) transport system including car travel and bus travel is studied in scenarios with or without the bus lane and passengers' in-vehicle comfort is considered, to 
propose cost function for both travel modes; (b) the game equilibriums of travel behavior for both car and bus travelers are analyzed to reveal their travel choice and relevant cost at system equilibrium; (c) Stackelberg model is built to describe the dynamic interactions between the government, the bus company, and travelers and to output the numerical analysis results at system equilibrium under the influence of different policy combination and resource allocation; (d) the kriging surrogate model based on system numerical simulation is constructed to get the solution of the game model and take minimizing TSC as the goal to seek for the optimal policy combination. The results show an effective performance that the optimized policy combination can reduce TSC index by $8.59 \%$ and $9.82 \%$ in $\mathrm{BL}$ scenario and NBL scenario, respectively, and reduce travel cost per person by $10.28 \%$ and $15.85 \%$ in BL scenario and NBL scenario, respectively.

This paper founds a basic model of the system to create the foundation for the future study. Travelers' heterogeneity can be taken into consideration to analyze different group of people with different travel preference. Besides, various aims of system optimization can be set to obtain Pareto optimal solution of policy and resource allocation based on multiobjective optimization. Finally, the algorithm for the model in the paper is an approximate global optimal algorithm based on the numerical simulation which needs to be improved by simplifying the model, deducing the analytical solution, and exploring the mechanism of how the policy can influence the transport system in the next step.

\section{Conflicts of Interest}

The authors declare that there are no conflicts of interest regarding the publication of this paper.

\section{Acknowledgments}

This work was supported by the National Natural Science Foundation of China under Grant no. 51338008 and no. 51278454 and the MOE (Ministry of Education in China) Project of Humanities and Social Sciences (17YJCZH124).

\section{References}

[1] W. Vickrey, "Congestion theory and transport investment," American Economic Review, vol. 59, pp. 251-261, 1969.

[2] H. Yang and H.-J. Huang, "Analysis of the time-varying pricing of a bottleneck with elastic demand using optimal control theory," Transportation Research Part B: Methodological, vol. 31, no. 6, pp. 425-440, 1997.

[3] W.-X. Wu and H.-J. Huang, "Equilibrium and modal split in a competitive highway/transit system under different road-use pricing strategies," Journal of Transport Economics and Policy, vol. 48, no. 1, pp. 153-169, 2014.

[4] W.-X. Wu and H.-J. Huang, "An ordinary differential equation formulation of the bottleneck model with user heterogeneity," Transportation Research Part B: Methodological, vol. 81, no. 1, pp. 34-58, 2015.

[5] E. J. Gonzales and C. F. Daganzo, "Morning commute with competing modes and distributed demand: user equilibrium, system optimum, and pricing," Transportation Research Part B: Methodological, vol. 46, no. 10, pp. 1519-1534, 2012.

[6] L.-L. Xiao, T.-L. Liu, and H.-J. Huang, "On the morning commute problem with carpooling behavior under parking space constraint," Transportation Research Part B: Methodological, vol. 91, pp. 383-407, 2016.

[7] M. Amirgholy and E. J. Gonzales, "Demand responsive transit systems with time-dependent demand: user equilibrium, system optimum, and management strategy," Transportation Research Part B: Methodological, vol. 92, pp. 234-252, 2016.

[8] E. DePalma and R. Arnott, "Morning commute in a single-entry traffic corridor with no late arrivals," Transportation Research Part B: Methodological, vol. 46, no. 1, pp. 1-29, 2012.

[9] C.-Y. Li and H.-J. Huang, "Morning commute in a singleentry traffic corridor with early and late arrivals," Transportation Research Part B: Methodological, vol. 97, pp. 23-49, 2017.

[10] P. G. Berglund and C. Kwon, "Solving a location problem of a Stackelberg firm competing with Cournot-Nash firms," Networks and Spatial Economics, vol. 14, no. 1, pp. 117-132, 2014.

[11] H. Wang, Q. Meng, and X. Zhang, "Game-theoretical models for competition analysis in a new emerging liner container shipping market," Transportation Research Part B: Methodological, vol. 70, pp. 201-227, 2014.

[12] H. J. Van Zuylen and H. Taale, "Urban networks with ring roads: Two-level, three-player game," Transportation Research Record, no. 1894, pp. 180-187, 2004.

[13] Y. Lim, B. G. Heydecker, and S. Lee, "A continuous network design model in stochastic user equilibrium based on sensitivity analysis," Journal of Advanced Transportation, vol. 39, no. 1, pp. 63-79, 2005.

[14] Y. Hollander, J. N. Prashker, and D. Mahalel, "Determining the desired amount of parking using game theory," Journal of Urban Planning and Development, vol. 132, no. 1, pp. 53-61, 2006.

[15] J.-S. Pang and M. Fukushima, "Quasi-variational inequalities, generalized Nash equilibria, and multi-leader-follower games," Computational Management Science, vol. 2, no. 1, pp. 21-56, 2005.

[16] Y. C. Ho, P. B. Luh, and R. Muralidharan, "Information structure, Stackelberg games, and incentive controllability," Institute of Electrical and Electronics Engineers Transactions on Automatic Control, vol. 26, no. 2, pp. 454-460, 1981.

[17] J. G. Wardrop, "Some Theoretical Aspects of Road Traffic Research," Operations Research, vol. 4, no. 4, pp. 72-73, 1953.

[18] M. J. Smith, "The existence of a time-dependent equilibrium distribution of arrivals at a single bottleneck," Transportation Science, vol. 18, no. 4, pp. 385-394, 1984.

[19] S. Song, Acuity, Crowding, Feature Detection, and FIXation in Normal and Amblyopic Vision, ProQuest LLC, Ann Arbor, MI, 2009.

[20] C. Audet, J. E. Dennis, D. W. Moore, A. Booker, and P. D. Frank, "A surrogate-model-based method for constrained optimization," in Proceedings of the 8th Symposium on Multidisciplinary Analysis and Optimization 2000, American Institute of Aeronautics and Astronautics, Long Beach,CA, USA, September 2000.

[21] X. Chen, L. Zhang, X. He, C. Xiong, and Z. Li, "Surrogate-Based Optimization of Expensive-to-Evaluate Objective for Optimal Highway Toll Charges in Transportation Network," ComputerAided Civil and Infrastructure Engineering, vol. 29, no. 5, pp. 359-381, 2014. 
[22] A. Keane, A. Forrester, and A. Sobester, Engineering Design via Surrogate Modelling: A Practical Guide, American Institute of Aeronautics and Astronautics, Inc., Washington, DC, 2008.

[23] X. Chen, M. Yin, M. Song, L. Zhang, and M. Li, "Social welfare maximization of multimodal transportation: Theory, metamodel, and application to Tianjin Ecocity, China," Transportation Research Record, vol. 2451, pp. 36-49, 2014.

[24] Li. Mian, Li. Genzi, and S. Azarm, "A Kriging Metamodel Assisted Multi-Objective Genetic Algorithm for Design Optimization," Journal of Mechanical Design, vol. 130, no. 3, pp. 405414, 2008.

[25] J. Liu, Z. Han, and W. Song, "Comparison of infill sampling criteria in kriging-based aerodynamic optimization," in Proceedings of the 28th Congress of the International Council of the Aeronautical Sciences 2012, ICAS 2012, pp. 1625-1634, Australia, September 2012. 


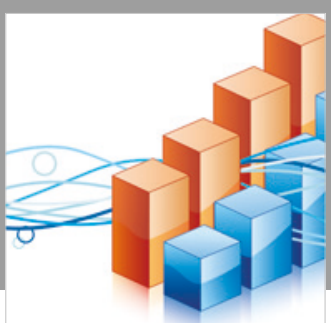

Advances in

Operations Research

\section{-n-m}
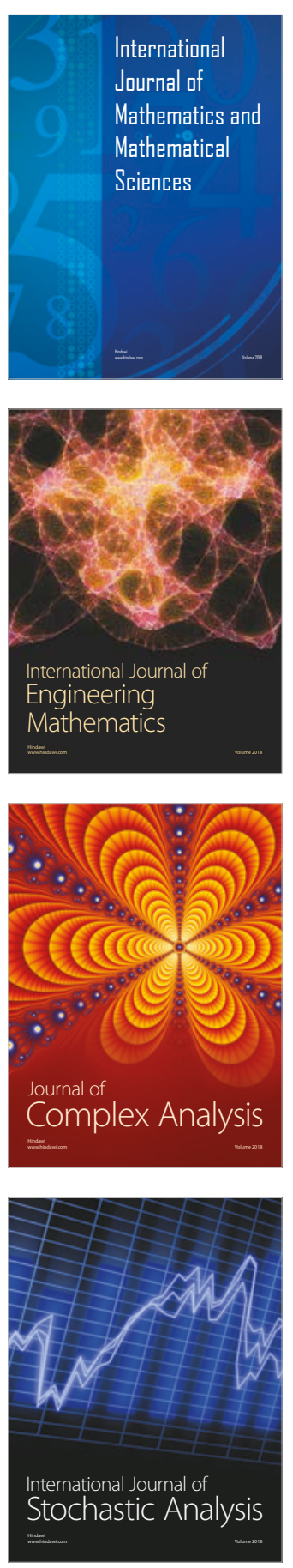
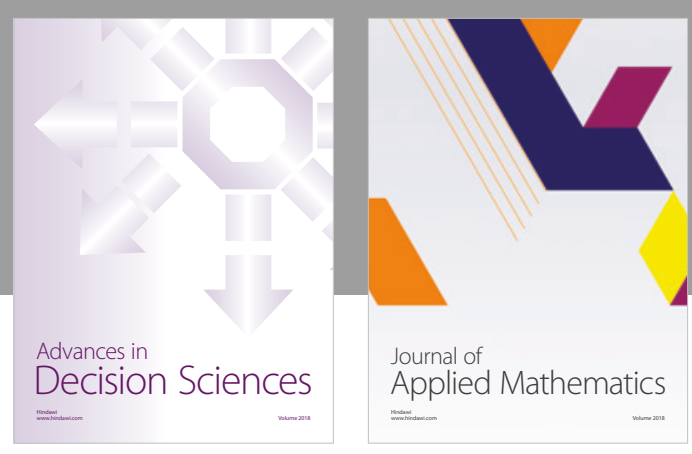

Journal of

Applied Mathematics
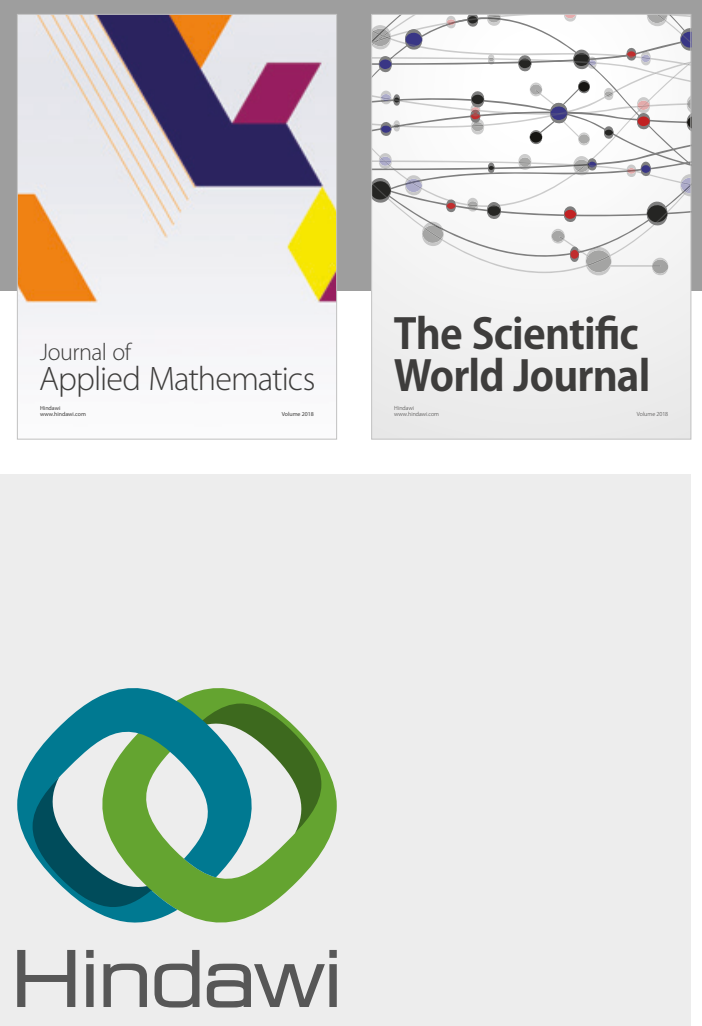

Submit your manuscripts at

www.hindawi.com

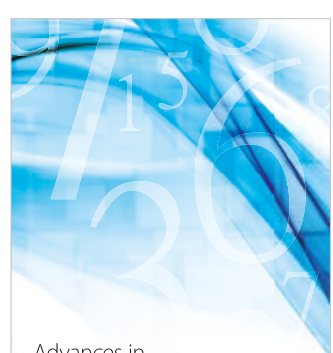

Advances in
Numerical Analysis
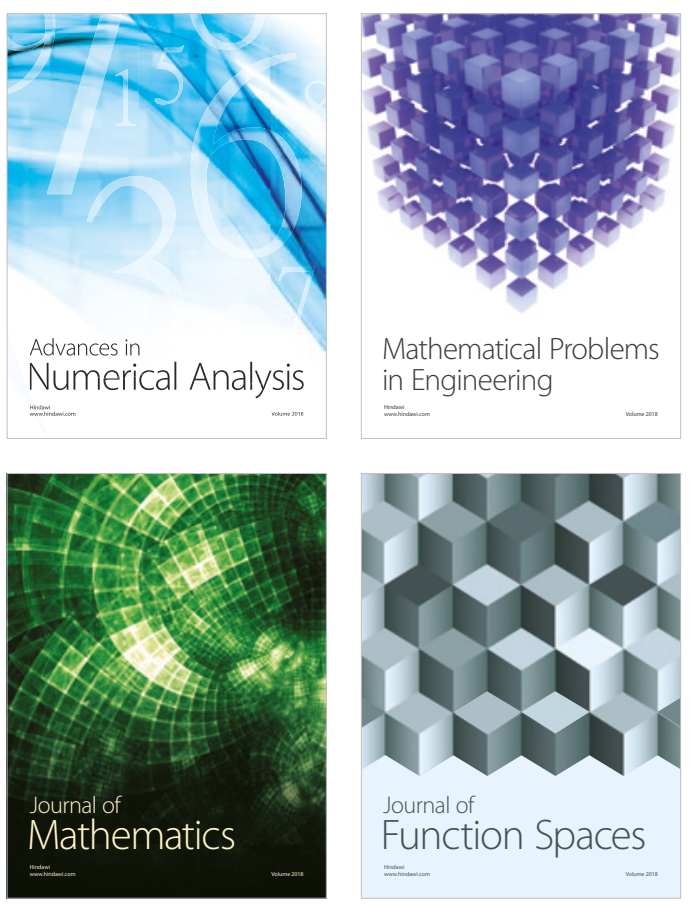

Mathematical Problems in Engineering

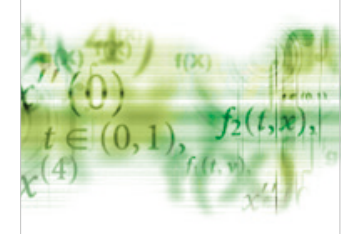

International Journal of

Differential Equations

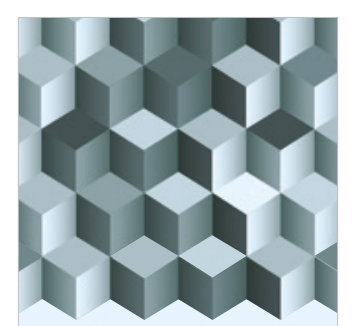

Journal of

Function Spaces

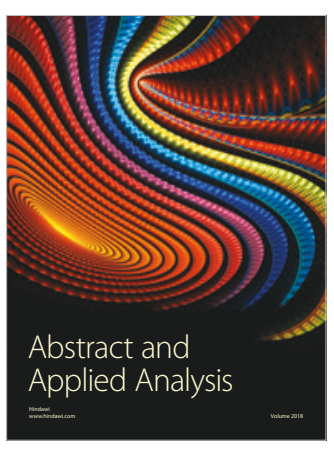

The Scientific

World Journal

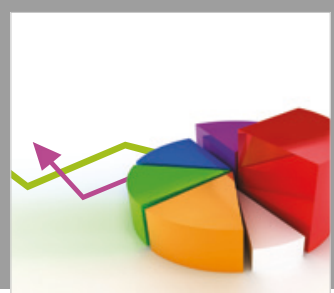

Journal of

Probability and Statistics
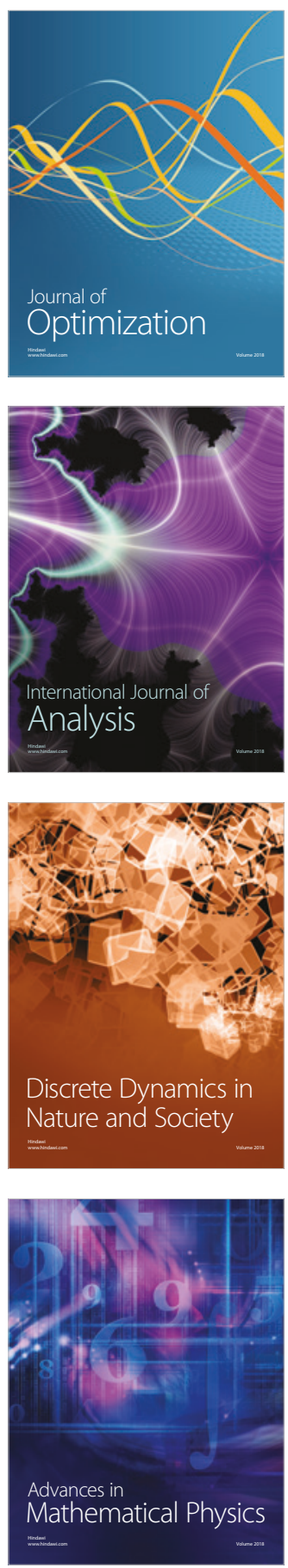